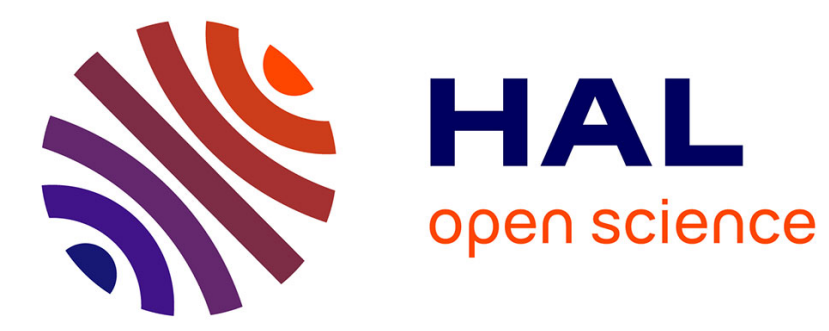

\title{
Optical quasicylindrical waves at dielectric interfaces
}

Choon How Gan, Loïc Lalouat, Philippe Lalanne, Lionel Aigouy

\section{To cite this version:}

Choon How Gan, Loïc Lalouat, Philippe Lalanne, Lionel Aigouy. Optical quasicylindrical waves at dielectric interfaces. Physical Review B: Condensed Matter and Materials Physics (1998-2015), 2011, 83, pp.085422. 10.1103/PhysRevB.83.085422 . hal-00679146

\section{HAL Id: hal-00679146 \\ https://hal-iogs.archives-ouvertes.fr/hal-00679146}

Submitted on 1 Dec 2015

HAL is a multi-disciplinary open access archive for the deposit and dissemination of scientific research documents, whether they are published or not. The documents may come from teaching and research institutions in France or abroad, or from public or private research centers.
L'archive ouverte pluridisciplinaire HAL, est destinée au dépôt et à la diffusion de documents scientifiques de niveau recherche, publiés ou non, émanant des établissements d'enseignement et de recherche français ou étrangers, des laboratoires publics ou privés. 


\title{
Optical quasicylindrical waves at dielectric interfaces
}

\author{
Choon How Gan, ${ }^{1}$ Loïc Lalouat, ${ }^{2}$ Philippe Lalanne, ${ }^{1, *}$ and Lionel Aigouy ${ }^{2}$ \\ ${ }^{1}$ Laboratoire Charles Fabry de l'Institut d'Optique, CNRS, Univ Paris-Sud, Campus Polytechnique, F-91127 Palaiseau Cedex, France \\ ${ }^{2}$ Laboratoire de Physique et d'Etude des Matériaux, ESPCI, CNRS UMR 8213 F-75231 Paris Cedex 5, France
}

(Received 6 October 2010; revised manuscript received 25 December 2010; published 25 February 2011)

\begin{abstract}
Much effort has been recently devoted to the study of the electromagnetic field launched on a metal-dielectric interface by one-dimensional (1D) subwavelength indentations. In this work, we consider the wave launched on a semiconductor surface that does not support any surface modes, in contrast to the metallic case. Through analytical calculations performed for polarized 2D Dirac line sources, we show that the waves launched by the two orthogonal polarizations (parallel and perpendicular to the surface) have approximately the same form, differing only in phase and magnitude. This finding implies that the wave launched on the surface by an arbitrary subwavelength indentation under an arbitrary illumination, either at grazing, normal, or oblique incidence, is always the same and possesses universal characteristics. We further observe this wave on a silicon substrate with a near-field probe. Through fitting with a simple model, the measured field is found to exhibit characteristics that are reminiscent of quasicylindrical waves on metal surfaces.
\end{abstract}

DOI: 10.1103/PhysRevB.83.085422

PACS number(s): 42.25.Fx, 78.67.-n

\section{INTRODUCTION}

The field scattered by subwavelength indentations in the vicinity of interfaces has been of long-standing interest in electromagnetism. In the 1900s, the rapid development of radio-wave technology prompted theoretical studies to explain why very long-distance (over-ocean transmission was achieved in 1907 by Marconi) transmission could be achieved with radio waves above the earth. The solution is indeed linked to guiding by the ionosphere layers, but at the beginning of the 20th century, the explanation was thought to be due to the nature of the surface waves launched on the flat earth by antennas. Sommerfeld was the first to determine the complete electromagnetic field radiated by a vertical dipole at the interface between two semi-infinite half spaces with permittivities $\epsilon_{m}$ and $\epsilon_{d}$. He verified that his complicated solution $^{1}$ is composed of a "direct contribution" and of a bounded Zenneck mode. ${ }^{2}$ The Zenneck mode, which is damped exponentially, is the analog of the surface plasmon polariton $^{3}$ (SPP) for metals at optical frequencies. On the other hand, the amplitude of the direct contribution does not decay exponentially but algebraically as $1 / r^{2}$ at asymptotically long distance from the antenna. ${ }^{4-7}$ This direct contribution, known as the Norton wave, was therefore believed to be responsible for long-distance radio transmission.

In nanophotonics, the field scattered by subwavelength indentations is also essential, since it is responsible for the electromagnetic interaction between nearby indentations on metallic surfaces. Most studies have focused on the SPPs, which have enabled researchers to overcome the diffraction limit for applications in microscopy,${ }^{8}$ nano-optical tweezing, ${ }^{9}$ integrated optics, ${ }^{10}$ and lasers. ${ }^{11}$ From a mathematical point of view, the solution of this photonic problem is identical to that of the radio-wave problem. ${ }^{12,13}$ However, there are also differences. We are mainly concerned by short-distance (rather than long-distance) electromagnetic interactions; the distance between two neighboring indentations in subwavelength optical surfaces is of the order of $\lambda$ and rarely exceeds $10 \lambda$. The second important difference concerns the fact that the dipole orientation cannot be chosen in nanophotonics. Focusing our discussion to line sources and transverse-magnetic (TM) polarization, two independent polarizations of the equivalent electrical dipole are generally excited with different strengths when any subwavelength two-dimensional (2D) indentation is illuminated.

Despite its importance for understanding the rich optics of subwavelength metallic surfaces, the field scattered by subwavelength indentations on a metal surface has been studied only recently. Lezec and colleagues ${ }^{14}$ were the first to emphasize the importance of a "direct" wave other than the SPP. This initial finding was, however, flawed by a misinterpreted model for diffracted evanescent waves, and has been followed by theoretical ${ }^{15-17}$ and experimental ${ }^{18}$ works aiming at determining the main characteristics of this wave. Importantly, it has been shown recently that the shape of this wave is almost independent of the polarization of the electrical line source. ${ }^{12,19}$ This implies that, besides the excitation of SPPs, any arbitrary subwavelength indentations illuminated by any incident electromagnetic field will additionally launch a unique field on a metallic surface. This field, which is therefore physically legitimate, is very different from the Norton wave for intermediate distances of interest $(x<10 \lambda)$, where it resembles a cylindrical wave. After some deliberations, ${ }^{15,16,18}$ this field has been referred to as a quasicylindrical wave (quasi-CWs) in the recent literature. ${ }^{12,19,20}$ Thus quasi-CWs on metal surfaces are, by definition, the fields scattered by subwavelength indentations on the surface, in addition to the SPP. ${ }^{12}$ In the present study, we are concerned by the field launched by a $2 \mathrm{D}$ subwavelength scatterer at the interface between two dielectric half spaces. The questions we try to answer are the same as those recently considered for metal interfaces: What is the field scattered on a dielectric interface? Is it independent of the incident illumination and can we therefore identify a physically legitimate wave similar to the quasi-CW at metallodielectric interfaces? If yes, what are the properties of this wave?

For that purpose, we investigate numerically and experimentally the characteristics of the fields scattered by a $2 \mathrm{D}$ subwavelength scatterer at the interface between two dielectric half spaces when it is illuminated by a wave polarized with 
a magnetic field along the scatterer (TM polarization). In Sec. II, we calculate the field radiated by a line source for the two orthogonal electric polarizations. It is found that the two source polarizations approximately launch the same identical wave on the surface (like for metal surfaces); this allows us to consider the launched wave as a unique entity that resembles a quasi-CW. Its physical existence is verified in Sec. III with experimental data collected through scanning near-field optical microscopy (SNOM) measurements for a metallic subwavelength ridge on a silicon substrate under oblique incidence illumination at $\lambda=975 \mathrm{~nm}$. The measured field is also shown to be consistent with fully vectorial numerical simulations obtained with an aperiodic Fourier modal method (a-FMM) ${ }^{21}$ Finally in Sec. IV, we offer concluding remarks.

\section{NUMERICAL ANALYSIS OF THE QUASI-CW}

To analyze the characteristics of the field launched on silicon-air interfaces and to derive general properties that should be shared by any subwavelength indentation, we consider the field radiated by a Dirac line source. Under illumination by an external TM-polarized electric field, $\mathbf{E}^{\mathrm{ext}}=E_{x}^{\mathrm{ex}} \hat{\mathbf{x}}+E_{z}^{\mathrm{ext}} \hat{\mathbf{z}}$, a small nonmagnetic $2 \mathrm{D}$ indentation can be approximately represented by two effective electric line sources (invariant in the $y$ direction), polarized either parallel or perpendicular to the interface, such that the induced polarization may be denoted as $\mathbf{p}=\left[p_{x}, p_{z}\right]$, with $p_{x}=\sigma_{x x} E_{x}^{\text {ext }}+\sigma_{x z} E_{z}^{\text {ext }}$ and $p_{z}=\sigma_{z x} E_{x}^{\text {ext }}+\sigma_{z z} E_{z}^{\text {ext }}, \sigma$ being a $2 \times 2$ polarizability tensor. Indeed the field generated by the two line sources with orthogonal polarizations generally differ. However, by analytically solving the problem of light emission by an oscillating electric line source on a metal-dielectric interface, ${ }^{12}$ it has been recently shown that the electromagnetic waves radiated by the $x$ - and $z$-polarized electric line sources on metallic surfaces are similar, provided that $\left|\epsilon_{m}\right| \gg \epsilon_{d}$ (a condition readily met in practice for metals illuminated below their plasma frequencies). We have verified that the same property holds for silicon surfaces. Figure 1 shows the field components of the waves generated by Dirac line sources (with unit amplitude), polarized either parallel $\left(p_{x}=1\right)$ or perpendicular $\left(p_{z}=1\right)$ to the interface, and located just above the interface (in air) at $x=0$. The calculation is performed using the rigorous formalism outlined in Ref. 12, with the refractive index of silicon $n_{\mathrm{Si}}$ taken to be 3.5. The plots on the left and the right sides give the field distribution for $\mathbf{p}=[1,0]$ and $\mathbf{p}=[0,1]$, respectively. Due to symmetry about the $x$ axis, only field distributions in the region $x>0$ are shown. The radiated field clearly has the nature of a cylindrical wave. Apart from a phase shift of approximately $180^{\circ}$, the waves generated by the two orthogonal polarizations strongly resemble each other. Qualitatively similar results are found for the field radiated by a polarized Dirac line source on a glass-air interface with $n_{\text {glass }}$ taken to be 1.5 .

It is clear that the waves generated by the $x$ - and $z$-polarized line sources differ in terms of their complex magnitude. However, they mainly differ in amplitude, but not in shape. By multiplying the response from the horizontal dipole by a complex factor $\sigma_{0}$, the normalized response, shown with red dots in Fig. 2, quantitatively matches the response obtained with the other polarization (black solid curve), the difference
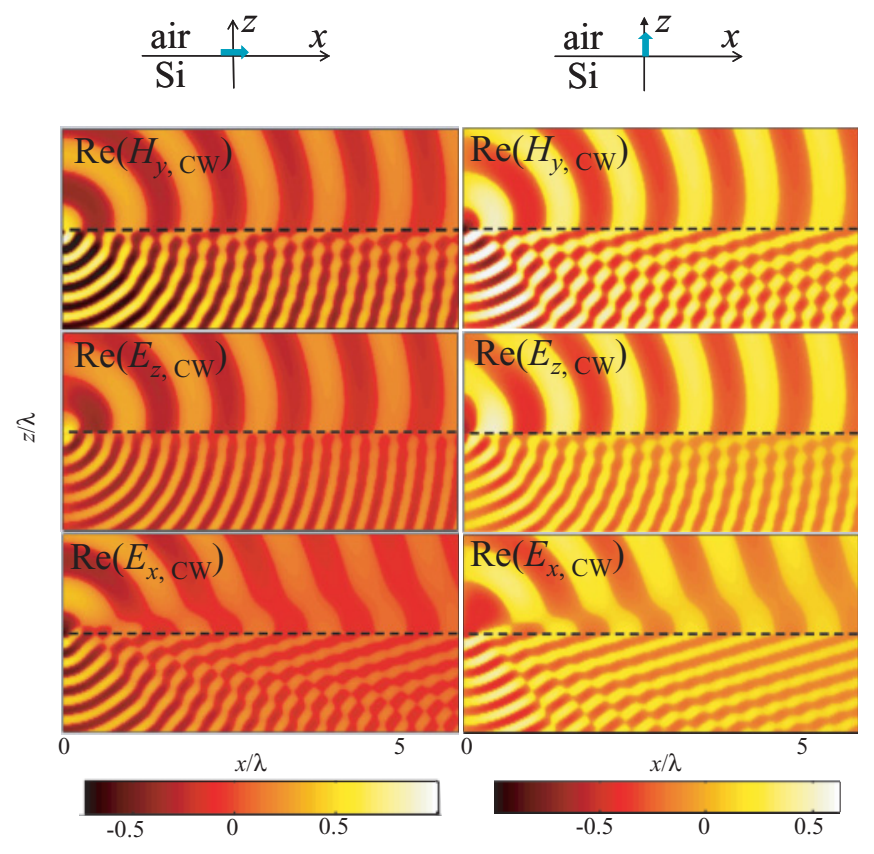

FIG. 1. (Color online) Electromagnetic field radiated by a Dirac line source at a silicon-air interface that is polarized either parallel to the interface along the $x$ direction ( $p_{x}=1$, left part), or along the $z$ direction perpendicular to the interface ( $p_{z}=1$, right part). The plots have been scaled nonlinearly to clearly show the field distribution near the interface. The schematic at the top of each column shows the polarization and position of the line source (thick blue arrow).

between the dots and the solid curve being almost invisible at the scale of the figure. This property is essential-in a nasty scenario, the two polarization responses would be different in shape implying that the field response of subwavelength indentations would strongly depend on factors such as their size and the angle of incidence of the external field, and no generic quasi-CW could be defined. The actual situation, as depicted in Fig. 2, is completely different. As for metallic interfaces, even if the polarizability tensor of subwavelength indentations strongly depends on the actual indentation geometry, size, and refractive index, the overall shape (not the complex amplitude) of the field scattered by an arbitrary subwavelength indentation illuminated by an arbitrary incident field may be regarded as a fixed quantity that we may refer to as a quasi-CW. This is the reason why, in Sec. III, a very specific incidence, imposed by the experimental apparatus constraints rather than any judicious choice, will be employed to observe the general properties of the quasi-CW. However, we note that differences, especially for the electric field, exist for small $x$, as seen in Figs. 2(b) and 2(c). These differences are much more distinct than for the case of the metallic surface. ${ }^{12}$ To better characterize the wave launched by the subwavelength indentation in the intermediate region $(x<10 \lambda)$, let us assume that the amplitude of the magnetic field component $H_{y, \mathrm{CW}}(x, z)$ at the dielectric surface $(z=0)$ takes on the form

$$
\begin{gathered}
H_{y, \mathrm{CW}}(x<0,0)=H_{0, \mathrm{CW}}^{(-)}(-x / \lambda)^{-m} \exp \left(-i k_{0} x\right), \\
H_{y, \mathrm{CW}}(x>0,0)=H_{0, \mathrm{CW}}^{(+)}(x / \lambda)^{-m} \exp \left(i k_{0} x\right),
\end{gathered}
$$




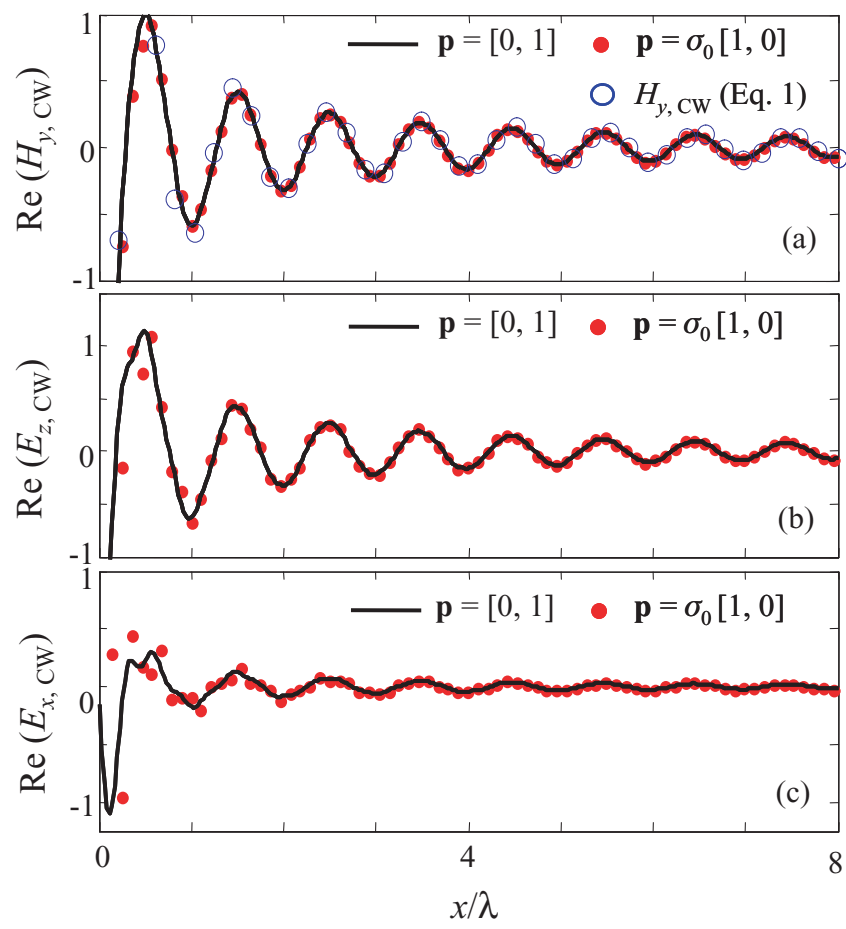

FIG. 2. (Color online) Illustrating the important fact that the fields launched on silicon-air interfaces by two orthogonal Dirac line sources differ only in amplitude but almost not in shape. The black solid lines represent the field components generated by a vertically polarized line source, $\mathbf{p}=[0,1]$, and the red dots represent the field components generated by a line source polarized parallel to the interface, $\mathbf{p}=\sigma_{0}[1,0]$. The complex parameter $\sigma_{0}=-3.71-i 0.67$ has been fitted for the best match between the black solid curves and the red dots. In (a), the close agreement with the analytic form of the launched wave given by Eq. (1) with $m=1$ is shown by the blue circles. A similar situation holds when the silicon substrate is replaced with a glass substrate $\left(n_{\text {glass }}=1.5\right)$. Note the discrepancy between the red dots and the solid curve for $E_{z}$ and $E_{x}$ for $x<2 \lambda$. This is not the case for metal-air interfaces (Ref. 12), for which a regular wavy nature is observed even for $x<2 \lambda$.

where $H_{0, \mathrm{CW}}^{( \pm)}$are constants, and $m$ is a damping exponent to be fitted. Recent theoretical studies have shown that this form is highly accurate for metallic-air interfaces. ${ }^{12}$ Fitting the approximate expression of Eq. (1) to the field launched at the interface by the two orthogonally polarized Dirac line sources, an excellent agreement is found for $m=1$, as shown by the blue circles in Fig. 2(a). While we have considered here a silicon substrate, we have checked numerically that Eq. (1) is valid for quasicylindrical waves launched on the surfaces of other dielectric or metal substrates at intermediate distances $(x<10 \lambda)$ provided that $\left|\epsilon_{\text {substrate }}\right| \gg\left|\epsilon_{d}\right|$, only the decay constant $m$ would be different.

For the sake of completeness, the decay rate of the field components of the quasi-CW as they radiate over long distances are shown in Fig. 3(a). For comparison, the case for the air-gold interface is shown in Fig. 3(b). Near the Dirac line source, the field components decay at slightly different rates. Nevertheless, they both initially decay at a rate close to $x^{-1 / 2}$, resembling a 2D cylindrical wave. For large distances, like the quasi-CW on metal-dielectric interfaces, the field propagating

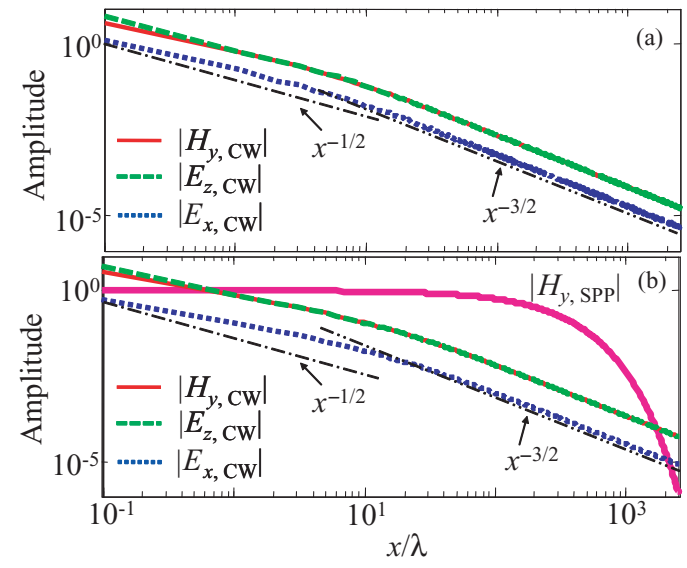

FIG. 3. (Color online) Decay characteristics of the field components of the wave launched at a silicon-air interface (a), and at a gold-air interface (b). The emitting line source is polarized along the $z$ direction normal to the interface, $\mathbf{p}=[0,1]$, and the refractive index of gold is $n_{\mathrm{Au}}=0.244+i 6.639$. Decay rates scaling as $x^{-1 / 2}$ (black dashed-dotted line) or as $x^{-3 / 2}$ (black dashed-dotted-dotted line) are added for reference.

on the silicon surface has a Norton-wave character, with a decay rate scaling as $x^{-3 / 2}$. For intermediate distances $(x<10 \lambda)$, the decay rate is between the two extremes $x^{-1 / 2}$ and $x^{-3 / 2}$. This is in agreement with the approximate form given by Eq. (1) for the launched wave, where the damping exponent $m$ is found to be close to unity. As will be seen in the next section, a similar value of $m$ is obtained by fitting the approximate form of Eq. (1) to the experimental data. It is to be noted that the line source has been taken to be polarized along $z$ in Fig. 3. Results with the line source polarized along $x$ are qualitatively similar.

\section{EXPERIMENT}

The experimental setup is shown in Fig. 4(a). The sample consists of a gold ridge (height $=120 \mathrm{~nm}$, width $=300 \mathrm{~nm}$ ) on top of a silicon substrate. It is illuminated by an intensity modulated laser diode at an angle of incidence $\theta \approx 73^{\circ}$ with operating wavelength $\lambda=975 \mathrm{~nm}$. The plane of incidence is perpendicular to the ridge and the polarization of the incident beam is in the plane of incidence, the so-called TM polarization. The near-field probe is a fluorescent erbium/ytterium codoped fluoride glass particle that absorbs the near-infrared photons, and re-emits light in the visible after a nonlinear absorption process. The measured fluorescence signal is proportional to the square of the intensity of the electromagnetic field. The particle, which has a size of $\sim 250 \times 250 \mathrm{~nm}^{2}$ is glued at the end of a sharp tungsten tip that enables topographic and optical measurements. ${ }^{22}$ As the length of the ridge $(>30 \mu \mathrm{m})$ is much longer than the wavelength, we will be primarily concerned with a $2 \mathrm{D}$ problem in the $x-z$ plane ( $y$ invariant, with $\Psi^{(\mathrm{TM})}=\left[E_{x}, H_{y}, E_{z}\right]$ ) instead of a full 3D problem to keep our analysis simple and intuitive.

The topography and the SNOM image collected for the gold ridge on the silicon substrate are shown in Figs. 4(b) and 4(c), respectively. On the side of the ridge nearer the laser source (i.e., $x<0$ ), rapidly varying interference fringes 

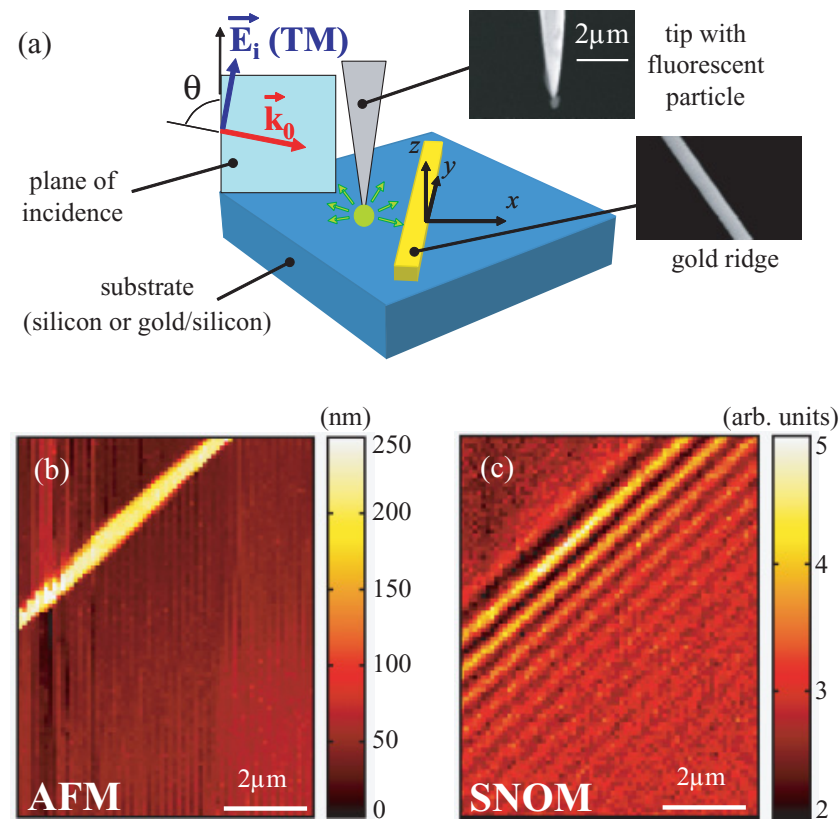

FIG. 4. (Color online) Description of the experiment. (a) Sketch of the experimental setup. (b) Topography of the ridge on the silicon surface. (c) SNOM image collected for the sample in (b).

are observed. These fringes arise as a result of interference between the incident field, the reflected field, and the wave launched by the ridge on the interface. The fringe period is $\Lambda=2 \pi /\left(k_{x}+k_{\mathrm{CW}}\right)$, where $k_{x}=k_{0} \sin \theta, k_{0}$ is the free-space wave-vector modulus, and $k_{\mathrm{CW}}$ is the wave number of the launched wave. Taking $k_{\mathrm{CW}}=k_{0}$ yields $\Lambda=\lambda /(1+\sin \theta) \approx$ $500 \mathrm{~nm}$, which is in good agreement with the measured data. It is noted that no fringes are observed when the incident beam is polarized with an electric field parallel to the ridge, evidencing that a wave is launched on the interface mainly for TM polarization. Calculations performed for a $2 \mathrm{D}$ line source indicate that the field scattered on the surface for TE polarization is not null; it rapidly decays over a couple of wavelengths. In addition, the SNOM signal is invariant along the $y$ axis, validating the $2 \mathrm{D}$ treatment of the problem. For distances within the vertical extent of the fluorescence particle $(\sim 250 \mathrm{~nm})$ along the $z$ axis, we have verified that the SNOM signal does not vary much across the surface of the silicon substrate, similar to what has been observed in Ref. 18 for metallic surfaces.

To analyze the SNOM data, we have fitted the experimental data with a simple analytical model where the total field is the sum of three components, namely the incident plane wave, the reflected plane wave, and the wave launched by the ridge [see Fig. 5(a)]. Using the approximate form of the quasi-CW [Eq. (1)] to represent the launched wave, let us express the total magnetic field on the side of the ridge nearer the laser $(x<0)$ at the interface $(z=0)$ as

$$
\begin{aligned}
H_{y}(x<0,0)= & H_{0}(1+r) \exp \left(i k_{x} x\right) \\
& +H_{0, \mathrm{CW}}^{(-)}(-x / \lambda)^{-m} \exp \left(-i k_{0} x\right),
\end{aligned}
$$

where $H_{0}$ is the complex amplitude of the incident magnetic field component, and $r$ is the Fresnel reflection coefficient of the flat interface. As will be seen shortly with fully vectorial calculations, the $z$ component of the electric field is dominant in the vicinity of the surface. Indeed, it can also be observed that $\left|E_{z}\right| \gg\left|E_{x}\right|$ in the previous section where the field radiated by the Dirac line sources was calculated (see Fig. 2). Neglecting the $x$ component of the electric field, the measured SNOM signal can be expressed $\mathrm{as}^{23}$

$$
S(x)=\int_{x-a / 2}^{x+a / 2}\left|E_{z}\left(x^{\prime}, 0\right)\right|^{4} d x^{\prime},
$$

where the convolution by the window $a$ is included to take into account the finite lateral extent $a$ of the fluorescence particle, and $E_{z}\left(x^{\prime}, 0\right)=i\left(k_{0} \epsilon_{0}\right)^{-1} \frac{\partial H_{y}}{\partial x^{\prime}}$, with $H_{y}$ as given in Eq. (2).

In Fig. 5(b), we compare the SNOM data (red circles) with the best fit obtained with Eq. (3) for $a=250 \mathrm{~nm}, m=1.1$, and $\left|H_{0, \mathrm{CW}}^{(-)} / H_{0}\right|=0.049$. The excellent agreement suggests that the field launched on the silicon surface by the ridge is well approximated by an analytical expression of the form given in Eq. (1). For the purpose of comparison, we have performed the same investigations for an identical ridge lying on a gold surface as shown in Fig. 5(c). In this case, the surface wave consists of a SPP and a quasi-CW. The presence of the SPP wave is taken into account in the model by adding the plasmon contribution, $H_{0, \mathrm{SP}}^{(-)} \exp \left(-i k_{S P} x\right)$, in Eq. (2), where $H_{0, \mathrm{SP}}^{(-)}$is the complex amplitude of the magnetic-field component of the launched SPP. The SNOM data are shown in Fig. 5(d) with red circles. The difference with the previous case is clear: The long-distance oscillations for the gold surface for $x<-4 \mu \mathrm{m}$ are mainly due to the interference of the SPP with the incident and the reflected field. Fitting $S(x)$ to the experimental data for the gold surface, it is found that the quasi-CW decays with a damping constant $m=1$, which is very close to the (a)

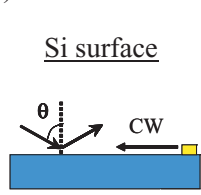

(b)

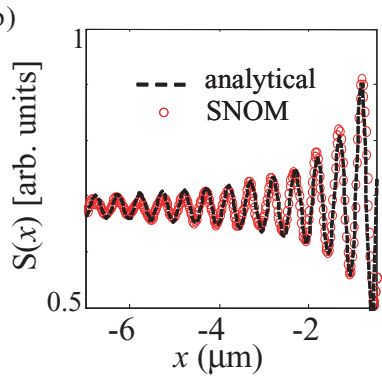

(c)

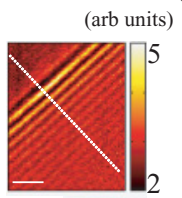

(d)

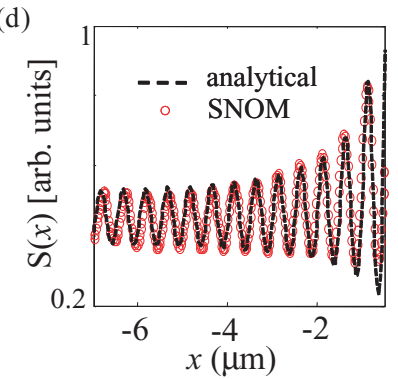

FIG. 5. (Color online) Comparison of the surface waves launched on silicon and gold surfaces. Experimental SNOM images measured on the silicon (a) and gold (c) surfaces. The white horizontal scale bar is $2 \mu \mathrm{m}$. Different types of surface waves launched by the ridges are shown according to the nature of the substrate. In (b) and (d), experimental data (red circles) collected along the cross-section indicated by the thick white line in (a) and (c) are fitted with results calculated from the analytical model (black dotted curve) for the case of the silicon and gold respectively. The maximum value of the SNOM data has been normalized to unity in the process of fitting. 


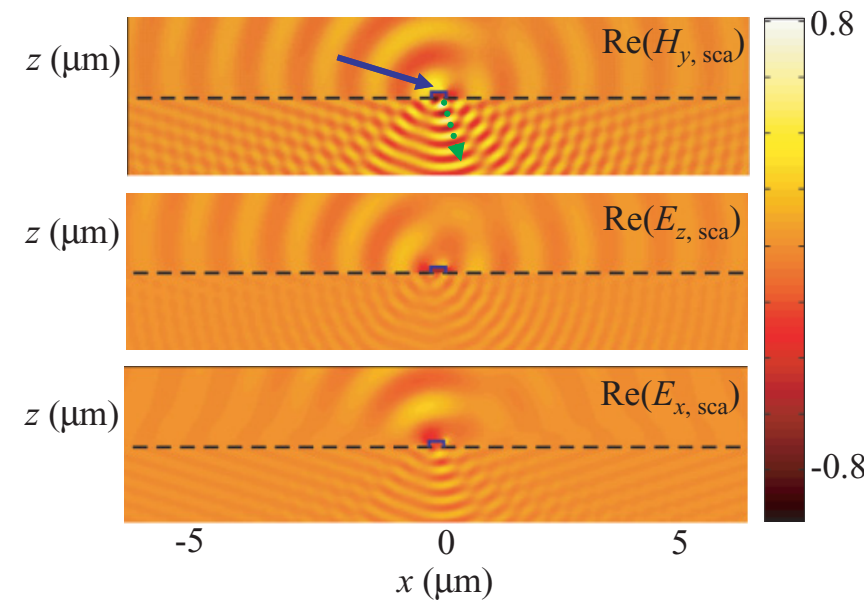

FIG. 6. (Color online) Electromagnetic field scattered by the ridge on top of the silicon slab. The solid blue arrow indicates the direction of the incident plane wave, and the dotted green arrow indicates the direction of the refracted plane wave. The scattered field is obtained by subtracting the plane waves, which are refracted and reflected at the planar interface, from the total field calculated with the a-FMM. The calculations are for an incident plane wave whose magnetic-field component is unity at $x=z=0$. The plots have been scaled nonlinearly to clearly show the field distribution near the interface.

value inferred for the silicon case, but is slightly larger than the expected values found in previous work. ${ }^{12}$ The ratios $\left|H_{0, \mathrm{CW}}^{(-)} / H_{0}\right|$ and $\left|H_{0, \mathrm{SP}}^{(-)} / H_{0}\right|$ for the case of the gold substrate are found to be 0.031 and 0.040 , respectively. For the two different substrates (silicon and gold), the excitation of the quasi-CW has the same order of magnitude, consistent with the field radiated by the unit amplitude Dirac line source shown in Fig. 3. Additionally, the SPP excitation at the wavelength of illumination $(\lambda=975 \mathrm{~nm})$ has the same order of magnitude as the quasi-CW excitation, which is also in agreement with calculations for the Dirac source.

To complete our discussion on the experimental results, we calculate the electromagnetic field $\Psi_{\text {sca }}=$ $\left[E_{x, \text { sca }}, H_{y, \text { sca }}, E_{z, \text { sca }}\right]$ scattered by the ridge on the silicon surface using the a-FMM method. The calculation is performed for an obliquely incident plane wave using a refractive index $n_{\mathrm{Au}}=0.244+i 6.639$ for gold, according to the data tabulated by Palik. ${ }^{24}$ As can be seen in Fig. 6, far from the interface the scattered field components resemble cylindricallike waves emanating from the ridge, either in air or in the silicon subtrate. However, near the interface the wave fronts of the continuous tangential components $\left(H_{y, \text { sca }}\right.$ and $\left.E_{x, \text { sca }}\right)$ exhibit strong distortions, which can be attributed to the large difference of the refractive index between silicon and air. Comparing to Fig. 1, each of the field components generated by the ridge or by the two Dirac line sources can be seen to share similar characteristics. The minor wave-front distortions close to the ridge are due to the finite size of the ridge, and are therefore not present in Fig. 1. The case with the gold surface shares similarities, but there are also major differences. For instance, due to the presence of the SPP, there is a horizontal plane located just above the air-gold interface where the magnetic-field component of the quasi-CW is nearly null. ${ }^{12}$ This is completely absent for the case of the silicon surface.

\section{CONCLUSIONS}

We have studied theoretically and experimentally the waves launched at the interface between a silicon substrate and air by a subwavelength indentation illuminated under TM polarization. It is worthwhile to note that the overall shape (not the amplitude modulus or phase) of the launched wave can be regarded as a unique physical entity that is independent of the incident illumination and of the actual geometry, size, and refractive index of the indentation. This property is fundamental, and is in fact the basis for attributing a quasi-CW characteristic to the launched field. Our analysis leads to a better understanding of the scattering phenomena of subwavelength indentations on planar interfaces, and could open up potential studies to exploit the characteristics of the quasi-CW in nanotechnologies.

\section{ACKNOWLEDGMENTS}

The authors thank M. Mortier for providing the fluorescent particles used in the near-field optical experiments, and J. P. Hugonin for assistance with the numerical analysis. L.L. and L.A. acknowledge the European Union (EU) for financial support through the project Nanomagma (Contract No. NMP3-SL-2008-214107). C.H.G acknowledges financial support from A*STAR of Singapore.

\footnotetext{
*Philippe.lalanne@institutoptique.fr

${ }^{1}$ A. Sommerfeld, Ann. Phys. (Leipzig) 81, 1135 (1926).

${ }^{2}$ J. Zenneck, Ann. Phys. (Leipzig) 23, 846 (1907).

${ }^{3}$ H. Raether, Surface Plasmons on Smooth and Rough Surfaces and on Gratings (Springer, Berlin, 1988).

${ }^{4}$ K. A. Norton, Nature (London) 135, 954 (1935).

${ }^{5}$ K. A. Norton, Proc. IRE 24, 1367 (1936); 25, 1203 (1937).

${ }^{6}$ R. E. Collin, IEEE Antennas Prop. Mag. 46, 64 (2004).

${ }^{7}$ A. Baños, Dipole Radiation in the Presence of a Conducting HalfSpace (Pergamon Press, Oxford, 1966).
}

${ }^{8}$ M. Specht, J. D. Pedarnig, W. M. Heckl, and T. W. Hänsch, Phys. Rev. Lett. 68, 476 (1992).

${ }^{9}$ M. Righini, A. S. Zelenina, C. Girard, and R. Quidant, Nature Phys. 3, 477 (2007).

${ }^{10}$ H. Ditlbacher, J. R. Krenn, G. Schider, A. Leitner, and F. R. Aussenegg, Appl. Phys. Lett. 81, 1762 (2002).

${ }^{11}$ M. A. Noginov, G. Zhu, A. M. Belgrave, R. Bakker, V. M. Shalaev, E. E. Narimanov, S. Stout, E. Herz, T. Suteewong, and U. Wiesner, Nature (London) 460, 1110 (2009). 
${ }^{12}$ P. Lalanne, J. P. Hugonin, H. T. Liu, and B. Wang, Surf. Sci. Rep. 64, 453 (2009).

${ }^{13}$ A. Y. Nikitin, S. G. Rodrigo, F. J. García-Vidal, and Martín-Moreno, New J. Phys. 11, 123020 (2009).

${ }^{14} \mathrm{G}$. Gay, O. Alloschery, B. Viaris de Lesegno, C. O'Dwyer, J. Weiner, and H. J. Lezec, Nature Phys. 2, 262 (2006).

${ }^{15}$ P. Lalanne and J. P. Hugonin, Nature Phys. 2, 551 (2006).

${ }^{16}$ L. Chen, J. T. Robinson, and M. Lipson, Opt. Express 14, 12629 (2006).

${ }^{17}$ A. Y. Nikitin, F. J. García-Vidal, and Martín-Moreno, Phys. Rev. Lett. 105, 073902 (2010).
${ }^{18}$ L. Aigouy, P. Lalanne, J. P. Hugonin, G. Julie, V. Mathet, and M. Mortier, Phys. Rev. Lett. 98, 153902 (2007).

${ }^{19}$ H. Liu and P. Lalanne, Phys. Rev. B 82, 115418 (2010).

${ }^{20}$ W. Dai and C. M. Soukoulis, Phys. Rev. B 80, 155407 (2009).

${ }^{21}$ J. P. Hugonin and P. Lalanne, J. Opt. Soc. Am. A 22, 1844 (2005).

${ }^{22}$ L. Aigouy, Y. De Wilde, and M. Mortier, Appl. Phys. Lett. 83, 147 (2003).

${ }^{23}$ B. Wang, L. Aigouy, E. Bourhis, J. Gierak, J. P. Hugonin, and P. Lalanne, Appl. Phys. Lett. 94, 011114 (2009).

${ }^{24}$ E. D. Palik, Handbook of Optical Constants of Solids (Academic, New York, 1985). 